\title{
Prediction of J-Integral dependence to residual stress and crack depth on NACA 0012-34 using FE and ANN
}

\author{
A.R. Hosseinzadeh ${ }^{*}$ and Mh. Karimi
}

Department of Mechanical Engineering, Bu-Ali Sina University, Hamedan, Iran

\begin{tabular}{|c|c|}
\hline ARTICLE INFO & A B S T RACT \\
\hline $\begin{array}{l}\text { Article history: } \\
\text { Received October 6, } 2014 \\
\text { Accepted } 2 \text { February } 2015 \\
\text { Available online } \\
2 \text { February } 2015 \\
\text { Keywords: } \\
\text { Residual stress } \\
\text { J-Integral } \\
\text { Crack } \\
\text { Finite element } \\
\text { ANN }\end{array}$ & $\begin{array}{l}\text { This paper presents an approach of linking finite element method with artificial neural network } \\
\text { to predict J-Integral parameter in desirable airfoil condition. Finite Element (FE) and Artificial } \\
\text { Neural Network (ANN) have been employed for the purpose. In other words, a prediction of } \\
\text { finite element results has been done using ANN. Ultimately results of two methods have been } \\
\text { compared for different cases. Wing fracture is a well-known problem of the planes which } \\
\text { depends on various parameters. The J-integral is a vital parameter in evaluations of structure } \\
\text { fracture phenomena. On the other hand residual stresses play an influential role in fracture } \\
\text { formation. In the current work, effect of residual stresses and crack depth on J-Integral has } \\
\text { been investigated in a standard NACA0012-34 airfoil. As will be seen, residual stresses and } \\
\text { crack depth influence J-Integral values. It also will be shown that predictions of ANN method } \\
\text { are in a good agreement with those obtained by finite element method. }\end{array}$ \\
\hline
\end{tabular}

(C) 2015 Growing Science Ltd. All rights reserved.

\section{Introduction}

Residual stresses play an influential role in many failure processes of engineering structures (Withers \& Webster 2001). If unknown, they can cause failure when combined with applied stresses (Totten, 2005). Residual stresses on engineering components which are produced during manufacturing are defined as internal loads. These loads along with other external loads can cause defects such as fatigue and stress corrosion cracking (Sherry et al., 2006). Process-induced residual stresses are inevitable for most mechanical or thermal operations used in processing engineering materials (Ren et al., 2009). Residual stresses are often generated by thermal gradient on material neighborhood of component parts. Various techniques exist for generating residual stress in engineering structures such as; shot peening, forging, quenching (Mahmoudi et al., $2012 \mathrm{a}, \mathrm{b}$ ) and welding. Loading and unloading is another one of them. In this study residual stresses have been generated on a model using the last

* Corresponding author

E-mail addresses: a.r.hosseinzade@basu.ac.ir (A.R. Hosseinzadeh) 
mentioned technique. Generally, using computer simulation clearly shows the characteristics and details of problems that are difficult, expensive or impossible to investigate experimentally. For accurate research the computational fluid dynamics (CFD) has been employed first. By simulation of air motion around the airfoil in balanced moving airplane, static pressure and velocity components on the airfoil boundaries can be obtained (Eleni et al., 2012).

In this work procedure, numerical study has two main steps and several petty steps. In the first step, static pressure and velocity components around the moving airfoil were obtained by numerical solving of the governing equations. This step was performed by the aid of Computational Fluid Dynamics (CFD) software of Fluent. In the second step, the data obtained from the first step was applied to a model of the airfoil built in mechanical analysis software of ABAQUS. For this application, two subroutines are required, DLOAD and DISP which are applicable in ABAQUS. In this model residual stress and crack seam modeling were performed and their influences over the J-Integral were investigated. These two main steps will be described more in next parts.

\section{Material and Airfoil}

In the current project, NACA-001234 airfoil has been developed. This airfoil was chosen because of its usage in many aircraft applications. Typical examples of this airfoil usage are in the B-17 Flying Fortress and Cessna 152. This type of airfoil is a symmetrical airfoil. It means that the top and bottom of the wing has similar curves. This type of airfoil is commonly used one on high performance, aerobatic aircrafts with a positive attach angle to generate reliable lift forces.

Pure aluminum has not enough strength to be used in aircraft construction. Today, various types of aluminum alloys are applied in constructions. Aluminum alloys are categorized by their major alloying component. The components which are more common for aluminum alloying are copper, magnesium, zinc and manganese. In the current study Al 5083 has been chosen as assigned material; the most important properties of this grade of aluminum alloy are good welding and corrosion resistance characteristics. After selecting the airfoil type and its material property, simulation of models has been carried out. In the next section, this operation will be described thoroughly.

\section{Numerical study}

As mentioned before numerical study of research has two main steps and several petty steps. In the first step which is done by FLUENT software, fluid motion around the airfoil is simulated. After fluid simulation, the static pressure and velocity components would be obtained. Then, in the second main step this data would be applied on a model in ABAQUS. For this application, two subroutines are required, DLOAD and DISP which are applicable in ABAQUS. In this model residual stress and crack seam are performed and their influence over the J-Integral is investigated. These two main steps will be described in the next parts.

\subsection{Computational Fluid Dynamics (CFD)}

Computational fluid dynamics (CFD) was employed in order to get more accurate results. Note that this step is not rather important alone in this study, because the main purpose of this work is investigating the residual stress and crack depth on the airfoil failure. In fact the influence of these parameters will superimpose with those generated by moving wing in air. Before process simulation, meshing was done in Gambit mesh generating software. No-slip condition was applied to the airfoil surface and Mach number was put equal to 0.9. Turbulent model of k- $\varepsilon$ was used and under relaxation coefficient was set rather low to be sure of solution convergence. By simulation of air motion around the airfoil in balanced airplane moving, static pressure and velocity components on the airfoil boundaries would be obtained. Fig. 1 shows contours achieved by numerical simulation of fluid flow 
around the airfoil. For every crack depth, this simulation must be done. As stated before, this data will be used in ABAQUS model in the next step.

After getting results, these data are applied to the ABAQUS model. An interpolation would be done on output data for agreement of the nodes data on Fluent and ABAQUS, first. Then subroutines would be written for applying parameters. DISP subroutine can be used to define the magnitudes of prescribed boundary conditions such as velocity and DLOAD can be used to define the variation of the distributed load magnitude (in this work static pressure) is as a function of position, time, element number, load integration point number, etc. In fact these two subroutines are used to apply FLUENT output as ABAQUS input.

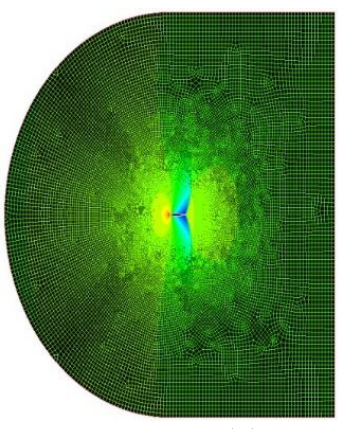

(a)
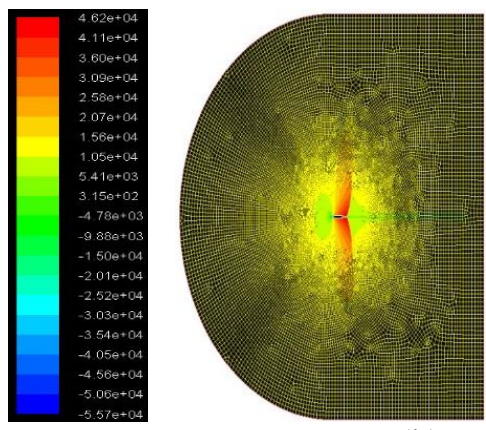

(b)

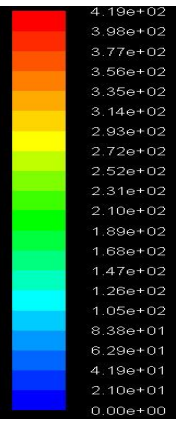

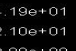

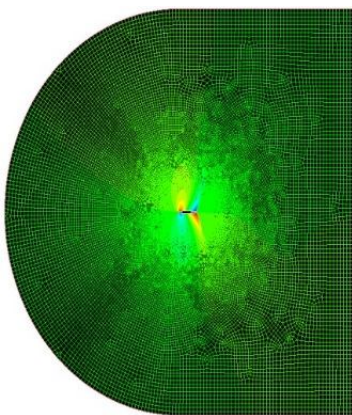

(c)

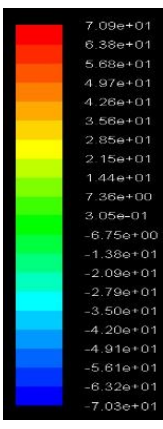

Fig. 1. Obtained contours in Mach number of 0.9 for (a) Static Pressure, (b) first component of velocity $\left(\mathrm{v}_{\mathrm{x}}\right)$, (c) second component of velocity $\left(\mathrm{v}_{\mathrm{y}}\right)$

\subsection{Finite Element}

In the current analysis, finite element method is used to simulate the airfoil in various situations. For all simulations of the current study, ABAQUS 6.10-1 has been employed. The ABAQUS simulation would be done after analyzing the flow over airfoils and writing the required subroutines. For simulation of airfoils, a deformable 2D planar and shell model is chosen for base feature. After modeling the NACA 0012-34 airfoil and assigning the Al 5083 properties on the model the solution steps must be performed. Three steps were taken for procedure completion. In the first step a static stage for applying written subroutines is established. The second and third steps include loading and unloading to produce residual stress on component. The level of residual stresses will be changed by variable loading on top edge of airfoil during the simulations. The range of this variance is 100-350 $\mathrm{MPa}$. On the other hand, crack length will change from 1 to $15 \mathrm{~mm}$. After completing the modeling and process simulation, meshing was done on model. CPE4R element type was applied to the model which is a 4-node bilinear plane strain quadrilateral, reduced integration, hourglass control. Meshing around crack must be finer to obtain suitable results. This operation is shown in Fig. 2 clearly. Note that mesh was checked exactly so that converged results become reliable and effect of elements size would be considered negligible.

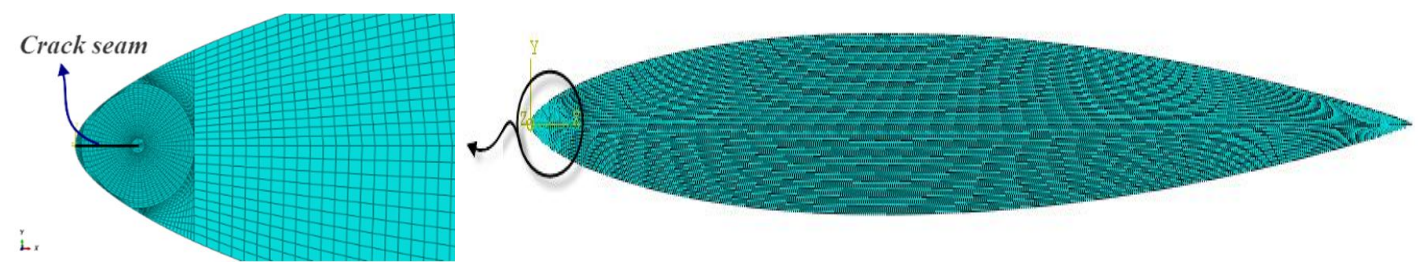

Fig. 2. A view of elements near crack seam 
After running the model, desirable results are obtained. For obtaining non-dimensional parameters, normalization process was done on achieved J-Integral values. Accordingly the obtained J- Integrals in all cases were divided to a defined parameter as $\mathrm{J}_{0}$. This definition is shown in Eq. (1);

$$
\mathrm{J}_{0}=\frac{\sigma_{\mathrm{y}}^{2}}{\mathrm{E}} \mathrm{a}_{\max }
$$

where $\sigma_{\mathrm{y}}$ is the yield stress, $E$ is Elasticity Modulus and $a_{\max }$ is the maximum crack length in current work that is equal to $15 \mathrm{~mm}$. Results are shown in Fig. 3 for all simulation cases.

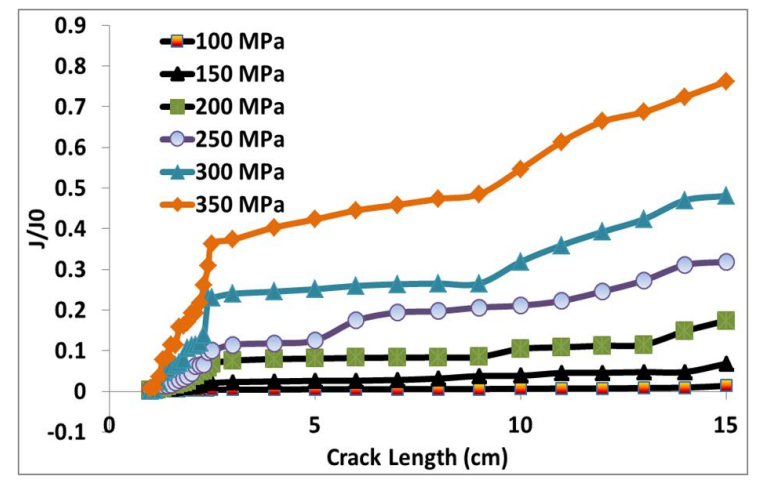

Fig. 3. J- Integral obtained in different levels of residual stress through finite element method

This Figure exactly shows the increase of J-Integral by increasing the crack length and residual stress levels. After completing the finite element procedure, some data must be taken as input data for artificial neural network in the next step.

\section{Artificial Neural Network}

Generally, the purpose of ANN (Artificial Neural Network) is to develop a mathematical structure that can be trained to obtain a set of outputs by a set of inputs by a mapping (Kim et al. 2004). ANN consists of the input layer, one or more hidden layers, and the output layer. Each layer consists of nodes or neurons. Each node has a transfer function (such as Sigmoid, Purlin, Hardlim, etc) activation function associated with it. The nodes in the hidden and output layers sum the weighted inputs from the sending nodes and apply this net input to the activation function. The output of the network is determined by applying the inputs and computing the output from the various nodes activations and interconnection weights (Al-Hailk et al., 2005).

Typically, in neural networks training, a particular input leads to a specific target output. Fig. 4 illustrates such a situation. In fact, the network is adjusted, based on a comparison of the output and the target, until the network output matches the target. Typically, sufficient such input/target pairs are needed to train a network. In practice, 80 percent of available data must be used for network training and the remaining 20 percent can be used to test of training neural network performance.

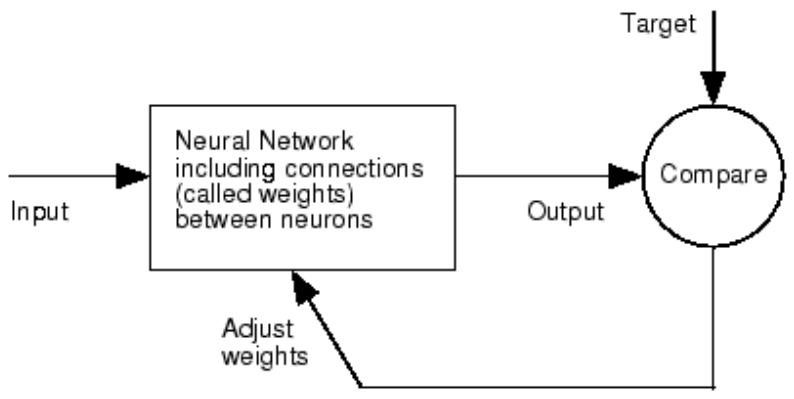

Fig. 4. ANN performance graph

To make an ANN, the first task is to determine the neural network topology, which includes the 
number of hidden layers, the number of neurons in each layer, transfer functions and the connections between them. The number of neurons in the input and output layers is governed by the dimensionality of the problem (Sirat \& Talbot, 2001).

In the current work, load and crack length are considered as input layer and J-Integral was taken as target/output. Input data for neural network training were imported from finite element results. In fact more finite element solutions has been performed in the out of current range of crack length an residual stresses until by use of them, ANN can predict the current target range.

For training neural network, two hidden layers considered which Sigmoid transfer function act in both layers. Network type was chosen as feed forward operation. Bias and weight factors which are applied for neural networks come in Table 1. For each case, a lot of attempts with different neuron numbers has been done, by comparing the obtained results by those achieved from finite element method, nearest network response was chosen as the best network which is seen in this Table. As shown in these tables, for each case, number of neurons, selected based on result, is exclusive.

Table 1.Bias and weight factors for designed Neural network in (a) $100 \mathrm{MPa}$, (b) $150 \mathrm{MPa}$, (c) $200 \mathrm{MPa}$, (d) $250 \mathrm{MPa}$, (e) $300 \mathrm{MPa}$, (f) $350 \mathrm{MPa}$.

\begin{tabular}{|c|c|c|c|c|c|c|c|c|c|}
\hline N. N & w1 & w2 & b1 & b2 & N. N & w1 & w2 & b1 & $\mathrm{b} 2$ \\
\hline \multirow{11}{*}{11 Neurons } & 15.5088 & 0.94505 & -15.2912 & -0.29845 & \multirow{11}{*}{10 Neurons } & 13.8239 & 0.005952 & -14.176 & -0.37836 \\
\hline & 15.3437 & 1.5579 & -12.3997 & & & 13.9145 & 1.1136 & -11.0093 & \\
\hline & 15.3605 & -0.01481 & -9.3021 & & & 13.9926 & 0.12508 & -7.7882 & \\
\hline & -15.4107 & -0.07099 & 6.1215 & & & -14 & 0.033242 & 4.6667 & \\
\hline & 15.3691 & 0.10479 & -3.1866 & & & -14 & -0.18677 & 1.5556 & \\
\hline & -15.4 & 0.11098 & -0.02663 & & & -13.9999 & 0.078182 & -1.5559 & \\
\hline & 15.4003 & 0.089372 & 3.0787 & & & -14.0028 & -0.11307 & -4.6563 & \\
\hline & -15.4002 & -0.03854 & -6.1583 & & & -14.0562 & 0.061566 & -7.6791 & \\
\hline & 15.4379 & 0.080349 & 9.1758 & & & 13.3571 & 0.51055 & 11.5763 & \\
\hline & -14.3195 & -1.0568 & -13.8835 & & & -14.2235 & -0.84929 & -13.7777 & \\
\hline & -15.8779 & -1.5174 & -15.3186 & & & & & & \\
\hline
\end{tabular}

(a)

(b)

\begin{tabular}{|c|c|c|c|c|c|c|c|c|c|}
\hline N. N & w1 & w2 & $\mathrm{b} 1$ & $\mathrm{~b} 2$ & N. N & w1 & w2 & b1 & $\mathrm{b} 2$ \\
\hline \multirow{11}{*}{11 Neurons } & 15.2067 & -0.11873 & -15.5933 & -0.13588 & \multirow{11}{*}{$\underline{8 \text { Neurons }}$} & 11.5091 & 1.9243 & -10.8937 & -0.30557 \\
\hline & 15.3999 & 0.82339 & -12.3201 & & & -11.4729 & -0.51136 & 7.6157 & \\
\hline & 15.4 & 0.51181 & -9.24 & & & 11.2011 & -0.12653 & -4.7936 & \\
\hline & -15.3996 & -0.21733 & 6.1613 & & & -11.2007 & -0.05427 & 1.6149 & \\
\hline & -15.3664 & -0.0482 & 3.19 & & & -11.0383 & -0.06932 & -1.7759 & \\
\hline & -15.3999 & -0.32331 & -0.16922 & & & -11.2529 & -0.19008 & -4.6538 & \\
\hline & -15.4 & 0.4844 & -3.0791 & & & 10.5907 & 0.43074 & 8.7406 & \\
\hline & 15.4 & 0.35586 & 6.16 & & & -11.1787 & -2.2099 & -11.4556 & \\
\hline & 15.4 & -0.005 & 9.24 & & & & & & \\
\hline & 14.8851 & 0.45413 & 12.8392 & & & & & & \\
\hline & -15.4335 & -1.0603 & -15.3664 & & & & & & \\
\hline
\end{tabular}

(c)

(d)

\begin{tabular}{|c|c|c|c|c|c|c|c|c|c|}
\hline N. N & w1 & w2 & b1 & b2 & N. N & w1 & w2 & b1 & b2 \\
\hline \multirow{8}{*}{$\underline{5 \text { Neurons }}$} & 7.0607 & 0.037096 & -6.9381 & 2.1752 & \multirow{8}{*}{$\underline{8 \text { Neurons }}$} & -11.9527 & -2.4751 & 10.4485 & 3.3193 \\
\hline & 5.9726 & 5.1361 & -4.9859 & & & 11.051 & 6.9782 & -8.2413 & \\
\hline & 6.8861 & 0.098659 & -0.96666 & & & -11.2602 & -0.66545 & 4.8299 & \\
\hline & 6.2637 & 0.3084 & 4.9865 & & & -11.1363 & -0.09449 & 1.2672 & \\
\hline & 6.0315 & 2.9159 & 6.7024 & & & -11.2 & 0.055278 & -1.5225 & \\
\hline & & & & & & -11.2004 & 0.092158 & -4.7994 & \\
\hline & & & & & & -10.7349 & -0.64267 & -8.3895 & \\
\hline & & & & & & -10.4567 & -6.6197 & -11.7593 & \\
\hline
\end{tabular}

(e)

(f)

Finally after applying bias and weight factors and network accomplishment, J-integrals, shown in Fig. 5 , are obtained. 


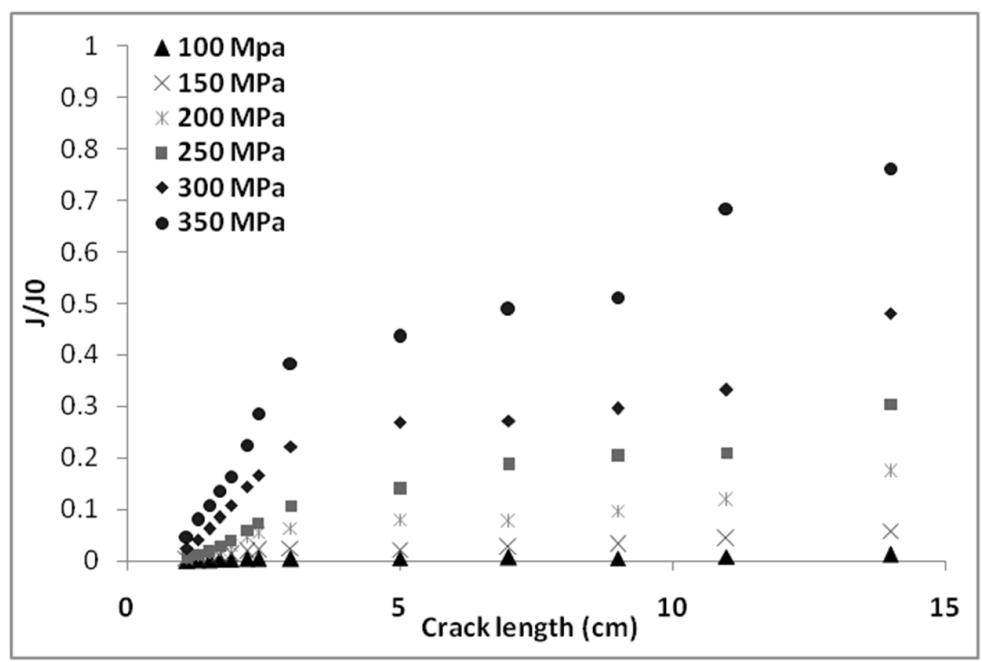

Fig. 5. Prediction of ANN for J-Integral in different levels of residual stress

Similar to finite element results, this Figure also shows increasing the J-Integral by the growth of crack length and residual stress but their agreement will be discussed in the next parts.

\section{Results}

In this part, prediction of ANN and those obtained by finite element have been compared in each case separately.

Fig. 6(a)-(f) shows this comparison. Each figure is for one residual stress level. This parameter has been increased in case (a) to (f) by the raise of applied load.

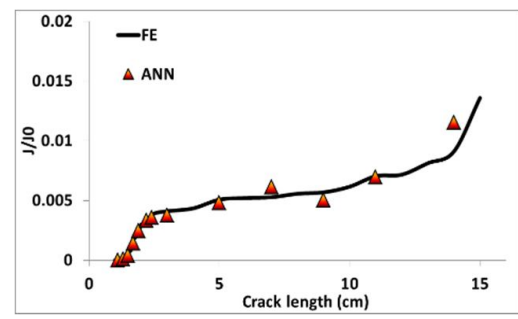

(a)

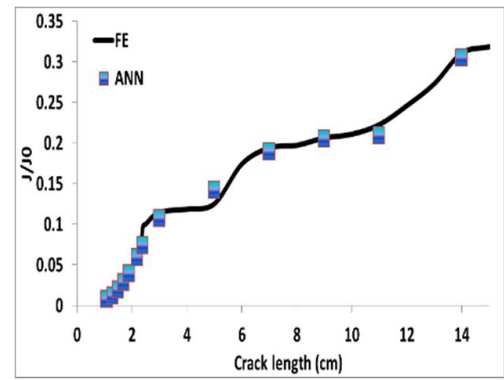

(d)

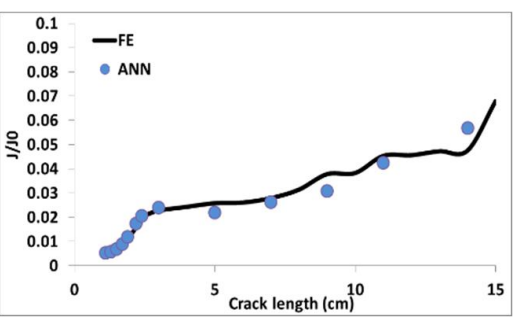

(b)

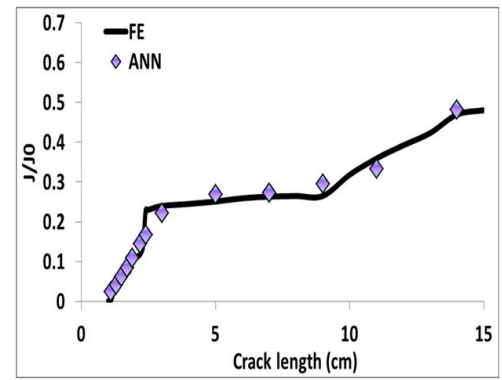

(e)

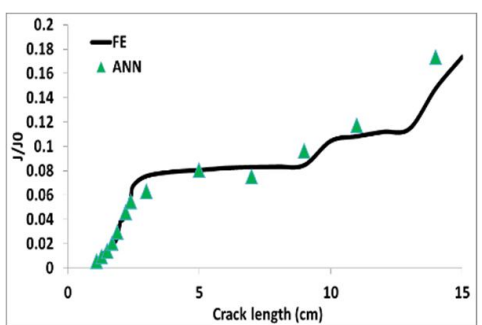

(c)

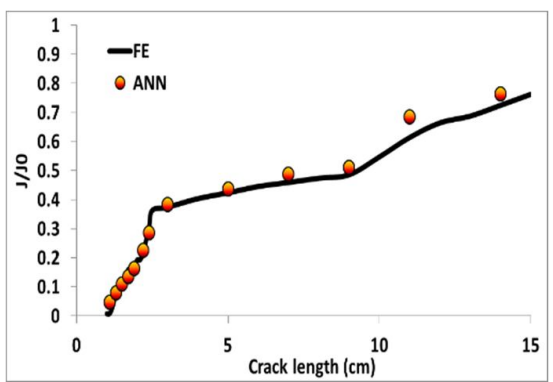

(f)

Fig. 6. Comparing the ANN predictions with those obtained through finite elements by a residual stress 
producer load equal to (a) $100 \mathrm{MPa}$, (b) $150 \mathrm{MPa}$, (c) $200 \mathrm{MPa}$, (d) $250 \mathrm{MPa}$, (e) $300 \mathrm{MPa}$, (f) $350 \mathrm{MPa}$

\section{Conclusion}

Residual stress plays a great role in many failure processes of engineering structures. In the current work a new approach the effect of residual stress and crack depth on J-Integral in a standard airfoil was studied using artificial neural network and finite element method. As shown in

Fig. 6, prediction of ANN has a good agreement with those obtained by the finite element method. This procedure is very efficient when development of results is required. Development of results means to obtain results for ideal state by design an ANN in certain state of data. This method especially is useful in cases which are not available or achieving desirable results may be difficult, problematic, expensive, etc. After designing an appropriate neural network, (assignment of hidden layers, neurons, and bias and weight factors) based on the correct data, it can be used for semi cases. This property is valuable and makes this method unique.

As shown in Fig. 3, 4 and 5, residual stress and crack depth are two determinant factors in J-Integral parameter. As observed, by the increase of residual stress, J-Integral increases too. This probably occurs because of increasing in plastic zone by addition in applied load for residual stress supplying. By increasing in residual stress producer load (and then unloading) more zones will be yield and this cause reduction in hardness and strength of component, thus by growth in residual stress, energy release rate will increases and this means increment in J-integral parameter. On the other hand it is clear that by the growth of crack depth, strength of component will decrease and energy release rate will increase and subsequently J-integral parameter will grow. These facts are shown in Figs. 3 and 4.

Concluding remarks of this work can be summarized as;

- $\mathrm{ANN}$ and FE predictions of J-Integral have a good agreement; it means that, the result of a suitable designed ANN can be used for prediction of finite element or other methods. in other words, a suitable neural network can be used as a crosscut which extends result in big desirable range.

- Growth in residual stress causes J-integral gaining, this means by increasing residual stresses in component the strength of it will be decreased and thus the energy release rate during crack growth will increase.

- J- Integral increases by crack growth. This is clear that crack growth leads to the decrease of the model strength and causes of increasing in energy release rate.

- All processes during manufacturing a component especially a vital component such as airplane wing must exactly be checked so that residual stress generation must be minimized. Because by residual stress growth, probability of failure would be increased sensitively.

\section{References}

Al-Haik, M. S., Hussaini, M. Y., \& Garmestani, H. (2006). Prediction of nonlinear viscoelastic behavior of polymeric composites using an artificial neural network. International Journal of Plasticity, 22(7), 1367-1392.

Eleni, D. C., Athanasios, T. I., \& Dionissios, M. P. (2012). Evaluation of the turbulence models for the simulation of the flow over a National Advisory Committee for Aeronautics (NACA) 0012 airfoil. Journal of Mechanical Engineering Research, 4(3), 100-111.

Kim, K. B., Yoon, D. J., Jeong, J. C., \& Lee, S. S. (2004). Determining the stress intensity factor of a material with an artificial neural network from acoustic emission measurements. NDT \& $E$ International, 37(6), 423-429.

Mahmoudi, A. H., Hosseinzadeh, A. R., \& Jooya, M. (2012a). Experimental calibration of the convection heat transfer coefficient in quenching process for a reliable prediction of residual 
stresses. In The International Conference on Experimental Solid Mechanics and Dynamics (XMech), Technology, U. o. S. a., Editor.

Mahmoudi, A. H., Hosseinzadeh, A. R., \& Jooya, M. (2012b). ). Experimental Measurement and Numerical prediction of Residual Stresses Using Contour Method in a Quenched Cylinder. In The International Conference on Experimental Solid Mechanics and Dynamics (X-Mech), Technology, U. o. S. a., Editor.

Totten, G., (2005), Handbook on residual stress, vol. 1. SEM, Bethel, ISBN: 978- 0871707291; p. 417.

Ren, X. B., Zhang, Z. L., \& Nyhus, B. (2009). Effect of residual stresses on the crack-tip constraint in a modified boundary layer model. International Journal of Solids and Structures, 46(13), 26292641.

Sherry, A. H., Da Fonseca, J. Q., Goldthorpe, M. R., \& Taylor, K. (2007). Measurement and modelling of residual stress effects on cracks. Fatigue \& Fracture of Engineering Materials \& Structures, $30(3), 243-257$.

Sirat, M., \& Talbot, C. J. (2001). Application of artificial neural networks to fracture analysis at the Äspö HRL, Sweden: fracture sets classification. International Journal of Rock Mechanics and Mining Sciences, 38(5), 621-639.

Withers, P. J., \& Webster, P. J. (2001). Neutron and synchrotron x-ray strain scanning. Strain, 37(1), 19-33. 